\title{
Safety of responsive neurostimulation in pediatric patients with medically refractory epilepsy
}

\author{
Fedor Panov, MD, ${ }^{1}$ Sara Ganaha, MD, ${ }^{1}$ Jennifer Haskell, PA, ${ }^{1}$ Madeline Fields, MD, ${ }^{2}$ \\ Maite La Vega-Talbott, MD, ${ }^{2}$ Steven Wolf, MD, ${ }^{2}$ Patricia McGoldrick, NP, MSN, MPA, ${ }^{2}$ \\ Lara Marcuse, MD, ${ }^{2}$ and Saadi Ghatan, MD ${ }^{1}$
}

\begin{abstract}
'Department of Neurosurgery, Mount Sinai West; and 'Department of Neurology, Icahn School of Medicine at Mount Sinai, New York, New York
\end{abstract}

OBJECTIVE Approximately $75 \%$ of pediatric patients who suffer from epilepsy are successfully treated with antiepileptic drugs, while the disease is drug resistant in the remaining patients, who continue to have seizures. Patients with drug-resistant epilepsy (DRE) may have options to undergo invasive treatment such as resection, laser ablation of the epileptogenic focus, or vagus nerve stimulation. To date, treatment with responsive neurostimulation (RNS) has not been sufficiently studied in the pediatric population because the FDA has not approved the RNS device for patients younger than 18 years of age. Here, the authors sought to investigate the safety of RNS in pediatric patients.

METHODS The authors performed a retrospective single-center study of consecutive patients with DRE who had undergone RNS system implantation from September 2015 to December 2019. Patients were followed up postoperatively to evaluate seizure freedom and complications.

RESULTS Of the 27 patients studied, 3 developed infections and were treated with antibiotics. Of these 3 patients, one required partial removal and salvaging of a functioning system, and one required complete removal of the RNS device. No other complications, such as intracranial hemorrhage, stroke, or device malfunction, were seen. The average followup period was 22 months. All patients showed improvement in seizure frequency.

CONCLUSIONS The authors demonstrated the safety and efficacy of RNS in pediatric patients, with infections being the main complication.

https://thejns.org/doi/abs/10.3171/2020.5.PEDS20118

KEYWORDS drug-resistant epilepsy; pediatrics; responsive neurostimulation

$\mathrm{E}$ PILEPSY is a common pediatric disorder affecting $1 \%$ of the population. ${ }^{1}$ Although children have an overall favorable long-term prognosis, roughly 10\%-30\% develop drug-resistant epilepsy (DRE), defined as "a failure of two or more anticonvulsant medications to achieve seizure freedom for a sustained period of time in either monotherapy or polytherapy."2-6 For patients with DRE, resection is indicated if the epileptic focus is identifiable and can be removed without compromising neurological function. Laser interstitial thermal therapy is an established, minimally invasive alternative to traditional resection with comparable clinical efficacy. ${ }^{7-9}$ Neither resection nor ablation is recommended in cases of multiple seizure foci, a focus in an eloquent area, or poor delineation of the network, a frequent occurrence in pediatric epilepsy. ${ }^{10-12}$ These limitations have led to the development of neuromodulatory treatments, such as vagus nerve stimulation (VNS) and deep brain stimulation (DBS). ${ }^{13}$ In 2013, the FDA approved the closed-loop responsive neurostimulation (RNS) system (NeuroPace Inc.) as an adjunctive therapy for adults with drug-resistant partial-onset seizures with no more than two epileptogenic foci. ${ }^{14-16}$

Only two case reports have demonstrated the efficacy of RNS in pediatric patients. ${ }^{17,18}$ In an effort to determine the safety and applicability of RNS in the pediatric group, we conducted a retrospective review of 27 consecutive cases

ABBREVIATIONS DBS = deep brain stimulation; DRE = drug-resistant epilepsy; $\mathrm{MDC}=$ multidisciplinary conference; MER = microelectrode recording; $\mathrm{MSHS}=$ Mount Sinai Health System; RNS = responsive neurostimulation; SEEG = stereo-EEG; VNS = vagus nerve stimulation.

SUBMITTED February 17, 2020. ACCEPTED May 4, 2020.

INCLUDE WHEN CITING Published online July 31, 2020; DOI: 10.3171/2020.5.PEDS20118. 
(age $<18$ years) with DRE that had undergone adjunctive treatment with RNS at our institution in the period from September 2015 to December 2019.

\section{Methods}

All surgical procedures were performed according to standard patient care. Patients and their families were informed of the proven efficacy of RNS in adults and the potential, yet unproven, therapeutic effects in the pediatric population. During such discussions, the off-label use of the device was made clear to the patients and their families, and viable alternatives were presented and discussed in full. We conducted a retrospective review of the electronic medical records (EMRs) with approval from the IRB of the Mount Sinai Health System (MSHS) to investigate surgical complications and postoperative seizure frequency.

\section{Study Variables}

Patient management was discussed at a multidisciplinary conference (MDC) consisting of neurosurgeons, epileptologists, neuroradiologists, neurologists, advanced practice providers, and neuropsychologists. Each evaluation followed the standards of our comprehensive epilepsy center with MRI, video-EEG, functional MRI, Wada testing, magnetoencephalography, and positron emission tomography, as indicated. Diagnostic intracranial EEG with either cortical grids/strips (4 patients) or stereo-EEG (SEEG; 23 patients) was performed, and a consensus was reached to rule out resective and ablative options at a follow-up MDC prior to RNS device implantation.

Seizure outcomes were reviewed using the EMRs, and outcome classification was recorded with a minimum follow-up of 1 year after RNS device implantation. In addition to applying Engel outcome classes, we stratified patients into quartiles according to the percentage reduction in seizure frequency as follows: group I, 75\%-100\% (including seizure freedom); group II, 50\%-74\%; group III, 25\%-49\%; and group IV, $0 \%-24 \%$ (including no improvement or worsening).

\section{Selection Criteria and Implantation Technique}

Pediatric patients with DRE who had been treated with RNS at the MSHS were eligible for inclusion in this study. We adopted the selection criteria and RNS implantation techniques previously discussed in adults. ${ }^{19}$

Targeting for RNS device implantation was performed on MRI volumetric sequences with gadolinium contrast using ROSA robotic software (Zimmer Biomet) for both depth and strip electrode placement. In the case of strip placement only, the StealthStation planning station (Medtronic) or intraoperative anatomical landmarks were used.

Implantations were planned considering the primary and alternative areas of stimulation. Placement of the ferrule, the tray for the neurostimulator, was based on skull thickness and ease of neurostimulator replacement or revision.

In cases requiring depth electrode placement, five bone fiducials were placed for registration (two at the coronal suture $4 \mathrm{~cm}$ from the midline, one anterior to the vertex midline, one at the vertex, and one in the parietal boss opposite the ferrule) with the patient under general anesthesia. An intraoperative volumetric CT or O-arm-acquired volumetric scan (Medtronic) was fused with preoperative MR images. Head pin fixation was performed using the Mayfield head clamp (Integra Life Sciences) or the Leksell base ring (Elekta), depending on exposure needs, and the head holder was directly connected to the robot. A satisfactory fiducial registration error (under $1 \mathrm{~mm}$ ) was confirmed in all cases. The head was prepped and draped in a sterile fashion, and cefazolin was administered in weightbased dosages intravenously within 1 hour of the incision, unless a documented penicillin allergy prompted the use of vancomycin.

If only strip electrodes were required, StealthStation facial registration was used for trajectory planning and placement. If the anatomy was well documented from an open diagnostic stage, anatomical landmarks (cortical veins and gyral patterns) and SEEG entry sites guided placement.

Minimal head shaving was performed to accommodate the ferrule incision and the smaller depth or strip electrode entry incisions. Depth electrodes were placed first to limit target error due to brain shift. Strip electrodes were subsequently placed utilizing loss of cerebrospinal fluid at the small dural opening at the ferrule site when possible. Intraoperative ECoG was done in all cases. All patients had a volumetric head CT after extubation to rule out malposition and hematoma as complications. All patients were observed for the 1st night in either the pediatric ICU or the neurosurgical ICU, and the majority were discharged home the next day. Neurostimulator replacement procedures- 6 cases to date-were performed for end-of-life devices by following the same paradigm.

\section{Data Analysis}

All patient data collected for the study were handled strictly by following the protocols approved by the IRB of the MSHS.

\section{Results}

\section{Patient Demographics}

Twenty-seven consecutive pediatric patients with DRE treated with RNS were included in this study (Table 1). The median age at implantation was 14 years (range 7-17 years). Over $40 \%$ (11 of 27) of patients had undergone previous resective surgery with continuing DRE at the time of MDC presentation at our center. Postoperatively, patients were closely followed by the comprehensive epilepsy team to evaluate for seizure frequency and surgical complications. The average follow-up was 22 months (range 9-50 months).

\section{Complications}

No postoperative hematoma, stroke, or RNS system malfunction was seen in our cohort. The percentages below are presented for both the total cohort $(\mathrm{N}=27)$ and those patients having reached a 1-year follow-up $(n=22)$. 
TABLE 1. Demographics of our pediatric RNS cohort, organized by relative success of outcomes

\begin{tabular}{|c|c|c|c|c|c|c|c|c|}
\hline $\begin{array}{l}\text { Case } \\
\text { No. }\end{array}$ & $\begin{array}{l}\text { Age at } \\
\text { Implant } \\
\text { (yrs) }\end{array}$ & $\begin{array}{c}\mathrm{SOZ} \\
\text { Identification* }^{*}\end{array}$ & $\begin{array}{l}\text { Implant } \\
\text { Strategy* }\end{array}$ & Primary Targets & $\begin{array}{l}\text { Alternative } \\
\text { Targets† }\end{array}$ & Prior Resection & $\begin{array}{c}\text { Outcome } \\
\text { Classification } \\
\text { (at 1-yr FU) }\end{array}$ & $\begin{array}{l}\text { Engel } \\
\text { Class }\end{array}$ \\
\hline 1 & 14 & SEEG & Depth & Lt SMA/It MTL & None & No & Seizure free & IIB \\
\hline 2 & 14 & SEEG & Strip & Lt OF & Lt temp & Lt ATLAH & $75 \%-99 \%$ & IB \\
\hline 3 & 17 & SEEG & Depth & Bilat inf parietal & ANT & No & $75 \%-99 \%$ & $\| \mathrm{B}$ \\
\hline 4 & 17 & SEEG & Combo & Lt OF & ANT & $\mathrm{CC}$ & $75 \%-99 \%$ & IIB \\
\hline 5 & 17 & SEEG & Combo & Rt OF/rt SMA & Rt temp & No & $75 \%-99 \%$ & IIB \\
\hline 6 & 17 & SEEG & Depth & Rt cing/lt insula & None & No & $75 \%-99 \%$ & IIB \\
\hline 7 & 12 & SEEG & Combo & CMPF & Bilat SMA & No & $75 \%-99 \%$ & IIB \\
\hline 8 & 10 & Grid \& strip & Strip & Lt medial M1/S1 & Lt lat M1/S1 & No & $75 \%-99 \%$ & IIIA \\
\hline 9 & 12 & Combo & Depth & Bilat ANT & Lt MTL/lt cing & Rt ATLAH & $75 \%-99 \%$ & IIIA \\
\hline 10 & 9 & SEEG & Depth & Rt PVNH/rt cing & Rt SMA/rt OF & Lt frontal disconnection & $75 \%-99 \%$ & IIIA \\
\hline 11 & 7 & SEEG & Strip & Rt medial M1/S1 & Rt lat M1/S1 & No & $75 \%-99 \%$ & IIIA \\
\hline 12 & 17 & Grid \& strip & Strip & Lt M1/S1 & Lt temp & Lt MST & $50 \%-74 \%$ & IIIA \\
\hline 13 & 12 & Combo & Depth & Lt CMPF/lt OF & Lt SMA & Rt hemispherec & $50 \%-74 \%$ & IIIA \\
\hline 14 & 10 & SEEG & Combo & Rt MTL/It ANT & Lt SMA/rt ANT & $\mathrm{CC}$ & $50 \%-74 \%$ & IIIA \\
\hline 15 & 9 & SEEG & Depth & Lt MTL/lt cing & Rt MTL & No & $50 \%-74 \%$ & IIIA \\
\hline 16 & 17 & SEEG & Depth & Bilat inf parietal & Bilat pst temp & No & $25 \%-49 \%$ & IIB \\
\hline 17 & 15 & Grid \& strip & Combo & ANT & Rt temp & $\begin{array}{l}\text { Rt frontal disconnection/ } \\
\text { CC/lt ATLAH }\end{array}$ & $25 \%-49 \%$ & IIIA \\
\hline 18 & 16 & SEEG & Combo & Lt MTL/lt insula & Lt SMA & No & $25 \%-49 \%$ & IIIA \\
\hline 19 & 15 & SEEG & Depth & S1 & Bilat frontopolar & Rt frontal resection & $25 \%-49 \%$ & IIIA \\
\hline 20 & 16 & SEEG & Depth & Bilat cing & Rt MTL & No & $25 \%-49 \%$ & IIIA \\
\hline 21 & 17 & SEEG & Depth & Bilat MTL & Bilat occ & No & $0 \%-25 \%$ & IVB \\
\hline 22 & 14 & SEEG & Combo & Rt OF/rt SMA & Bilat ANT & No & $0 \%-25 \%$ & IIIA \\
\hline 23 & 12 & Combo & Depth & Rt inf parietal/rt superior parietal & None & No & NA & NA \\
\hline 24 & 9 & SEEG & Depth & Lt CMPF/rt frontal & Lt MTL/rt CMPF & $\mathrm{CC}$ & NA & NA \\
\hline 25 & 17 & SEEG & Depth & Lt PVNH/lt MTL & None & No & NA & NA \\
\hline 26 & 16 & SEEG & Depth & Rt insula & None & $\begin{array}{l}\text { Rt ATLAH/rt frontal discon- } \\
\text { nection }\end{array}$ & NA & NA \\
\hline 27 & 17 & SEEG & Strip & Lt frontal//t temp & None & No & NA & NA \\
\hline
\end{tabular}

ANT = anterior nucleus of the thalamus; $\mathrm{ATLAH}=$ anterior temporal lobectomy with amygdalohippocampectomy $\mathrm{CC}=$ corpus callosotomy; cing = cingulate; $\mathrm{CMPF}=$ centromedian parafascicular nucleus; Combo = combination; FU = follow-up; hemispherec = hemispherectomy; inf = inferior; $\mathrm{MST}=$ multiple subpial transections; $\mathrm{MTL}$ $=$ mesial temporal lobe; $\mathrm{M} 1=$ primary motor; $\mathrm{NA}=$ not available; $\mathrm{No}=$ no surgery; $\mathrm{occ}=$ occipital; $\mathrm{OF}=$ orbitofrontal; pst $=$ posterior; $\mathrm{PVNH}=$ paraventricular nodular heterotropia; $\mathrm{SMA}=$ supplementary motor area; $\mathrm{SOZ}$ = seizure onset zone; $\mathrm{S} 1$ = primary sensory; temp = temporal.

* The term "combo" (combination) indicates that both grids and strips as well as SEEG were used, but the electrodes were not necessarily implanted during the same surgery.

† "Alternative targets" refer to the areas implanted but not connected for possible reattachment in the future.

Three patients (11\%) in our cohort experienced postoperative infections, with 2 (7\%) requiring return to the operating room and 1 (4\%) requiring complete explantation of the system (Table 2). A 14-year-old male developed a superficial infection at a small strip electrode incision 2 months after RNS device placement. The patient was seen continuously picking at the incision. He was conservatively treated with antibiotics along with outpatient incisional care. The infection resolved completely without surgical intervention. He is now 28 months post-RNS device placement and seizure free.

In another case, a 17-year-old male developed an infection at a depth incision contralateral to the ferrule. This patient noticed drainage 6 weeks after RNS system implantation, was admitted to the hospital, and had two at-risk depth electrodes removed and the small incisions washed out. The other two depth electrodes, ferrule, and neurostimulator were salvaged. He was discharged the same day. Treatment with antibiotics resolved his infection, and he continued to have seizure reduction with the two remaining depth electrodes.

In the third case, an 18-year-old male (17 at the time of the implant) experienced a delayed infection 4 months after the RNS system placement. After a trial of antibiotics, the entire system was explanted because of continuing drainage from the incision. The patient was doing well 6 months after explantation, with a planned reimplantation being considered by the family. 
TABLE 2. Overview of postoperative complications from RNS device implantation in a pediatric cohort

\begin{tabular}{lcl}
\hline \multicolumn{1}{c}{ Complication } & Events/Total Patients & $\begin{array}{c}\text { Events/Patients } \\
\text { w/ 1-Yr FU }\end{array}$ \\
\hline Infection & $3 / 27(11 \%)$ & $3 / 22(14 \%)$ \\
\hline Intracranial hemorrhage & $0 / 27$ & $0 / 22$ \\
\hline Stroke & $0 / 27$ & $0 / 22$ \\
\hline Device malfunction & $0 / 27$ & $0 / 22$ \\
\hline Device migration & $0 / 27$ & $0 / 22$ \\
\hline
\end{tabular}

\section{Clinical Outcomes of RNS}

Patients had regular follow-up visits with their epileptologists and neurosurgeons postoperatively. Twenty-two of the 27 patients had at least a 1-year follow-up. The breakdown of seizure reduction was as follows: 11 had a $75 \%-100 \%$ reduction, 4 had a $50 \%-74 \%$ reduction, 5 had a $25 \%-49 \%$ reduction, and 2 patients experienced a $0 \%-$ $24 \%$ reduction (Table 3 ). No patient suffered a worsening of seizure control.

\section{Discussion}

FDA approval of RNS for adjunctive treatment of DRE heralded a paradigm shift in neuromodulation for epilepsy. The ability to monitor and modulate seizures on a long-term, outpatient basis offered a way forward to a subset of patients without a valid alternative. RNS has several advantages over the more invasive treatment methods such as resection or ablative therapies. RNS is reversible, can be modified over time, and does not preclude other surgical treatments if the gathered long-term data open up such a possibility. Unlike resective and ablative therapies, whose efficacy decreases with time, RNS effectiveness improves with time, possibly because of a chronic neuromodulatory effect. ${ }^{11,20}$ While the RNS device requires a full-thickness craniotomy, patients tolerate this well without chronic discomfort. Some advantages of an implanted RNS system compared with the DBS experience in the neurosurgical community include its lack of moving parts, the absence of pulling (bowstringing) of the extensions, and lack of additional chest incisions. These characteristics could decrease the hardware failure rate seen in DBS, as issues such as extension fractures and twirling of the pulse generator are eliminated. Approval of the implanted RNS device for MRI in early 2020 is an important step in allowing utilization of the device in cases in which frequent MRI scans are required.

RNS allows for chronic monitoring in an ambulatory setting, overcoming temporal restrictions and pharmacological perturbations of the epilepsy monitoring unit (EMU). ${ }^{21,22}$ The seizure burden can be more accurately obtained, as seizures can be subclinical. Children tend to have less clear localization on EEG and semiology, making it difficult to pinpoint epileptogenic foci. ${ }^{23}$ The children's "immature" central nervous system may contain diffuse epileptogenic networks. ${ }^{24}$ McGovern et al. showed that despite SEEG defining an epileptogenic zone in $95 \%$ of cases, only $50 \%$ seizure freedom was achieved with
TABLE 3. Outcome classification based on percent decrease in seizure frequency, classified into quartiles

\begin{tabular}{cc}
\hline $\begin{array}{c}\text { Outcome Classification (\% decrease in } \\
\text { seizure frequency) }\end{array}$ & $\begin{array}{c}\text { No. of } \\
\text { Patients (\%) }\end{array}$ \\
\hline Group I: $75 \%-100 \%$ & $12(55)$ \\
\hline Group II: $50 \%-74 \%$ & $4(18)$ \\
\hline Group III: $25 \%-49 \%$ & $4(18)$ \\
\hline Group IV: $0 \%-24 \%$ & $2(9)$ \\
\hline
\end{tabular}

Twenty-two patients with a postoperative follow-up of at least 1 year were evaluated.

therapeutic stages because of the spread of electrical activity from an untreated location in the complex circuit. ${ }^{23}$ Pediatric diagnostic stages may be less accurate in epileptic focus localization, leading to less effective ablation or resection. ${ }^{25}$ RNS may improve the evaluation of pediatric epilepsy networks outside the EMU setting, as reported in adults. ${ }^{21}$

Given the initial promising RNS experience in adults, our center initiated off-label placement of the RNS device in the pediatric population as a palliative option for patients and families who had been through numerous diagnostic and therapeutic stages. Philosophically, our center embraced the idea by Gillis and Loughlan: "Both children and adults belong to the human race. Progress in medicine can be applied to and be of benefit to all. The transition from childhood to adulthood should be one of continuity rather than migration." 26 In each case, the patient and family were thoroughly counseled about the procedure and the lack of current evidence to support efficacy. Alternatives, including no intervention and VNS, were also presented and discussed.

Off-label implantation is difficult in part because of the resistance of insurance companies. While a significant minority of our patients on Medicaid or combined Medicaid/Children's Health Insurance Program (CHIP) saw no hurdles in preapproval, the majority of patients with private insurance required significant effort and frequent communications with medical directors (peer to peer) and even several specialty peer-to-peer phone reviews with outside neurosurgeons. Several insurance companies such as Aetna and Blue Cross Blue Shield are notoriously difficult, and we attempted to preempt denials by sending SEEG data, seizure diaries, studies linking improved seizure control to future cost savings, ${ }^{27}$ and letters from providers and family for justification.

Epilepsy surgery is still underutilized across the pediatric and adult populations. The ability to offer a reversible or modulatory therapy can help to address the fear that ablative or irreversible approaches can cause. This may alleviate anxiety and aid in parental decision-making.

\section{Outcomes}

The Engel scale was designed to measure the degree of seizure reduction or the achievement of seizure freedom after resective surgery. However, RNS is an adjunctive treatment designed to detect and abort a seizure after its onset. Therefore, judging RNS by a scale focused on sei- 
zure freedom is difficult. In addition to applying the Engel scale, we evaluated the decrease in seizure frequency. There was a significant decrease overall, with over half of the patients experiencing a $75 \%$ or more reduction in seizure frequency (Table 3). No patient had worsening of seizures after RNS system implantation.

RNS efficacy improves with each year the implant is active. ${ }^{11,20}$ Hence, reporting the Engel classification and reductions in seizure frequency in the early postimplant phase likely underestimated its eventual therapeutic effect. Nevertheless, the majority of the patients already showed improvement at 1 year.

\section{Complications}

To our knowledge, to date there are no large-scale pediatric RNS studies. Currently, the closest comparison groups include pediatric DBS, grid/strip implants, and SEEG. In such studies, bleeding, infection, and device malfunction were among the major contributors to morbidity. ${ }^{28}$

\section{Hemorrhage}

Bleeding is among the most feared complications in neuromodulation. In McGovern and colleagues' series of 64 robot-assisted SEEG implantations in children, ${ }^{23} 1$ patient $(1.6 \%)$ suffered major intraparenchymal hemorrhage requiring hematoma evacuation and electrode removal. In a large review of DBS for Parkinson's disease, hemorrhage was among the most common early complications ( $<90$ days postsurgery) at $1.4 \% .{ }^{29}$ In microelectrode recording (MER)-guided DBS studies, the risk of symptomatic hemorrhage was $0.5 \%-2.2 \%,{ }^{30,31}$ while studies using interventional MRI-guided DBS for pediatric dystonia did not show hematoma as a complication. ${ }^{32}$ Postoperative hematoma rates in pediatric strip and grid cases have ranged from $0 \%$ to $0.8 \% .^{33,34}$

\section{Infection}

Infection can be a major challenge with hardware implantation. Arya et al., who reported adverse events after invasive EEG grid/strip monitoring in 2542 patients, found that the most common complications were neurological involvement (2.3\%) and superficial infections $(3.0 \%) .{ }^{28}$ Pediatric VNS infection rates have ranged from $3 \%{ }^{35}$ (all requiring reoperation) to $9 \%$ (5\% requiring reoperation). ${ }^{36}$ The Stimulation of the Anterior Nucleus of the Thalamus for Epilepsy (SANTE) trial, the most appropriate adult comparison group receiving DBS for epilepsy, reported a $12.7 \%$ infection rate with the need for surgical explantation (partial or total) in $7 \% .{ }^{37}$ By comparison, the infection rate in RNS in adults has ranged up to $9 \%$, and surgical revision was required in up to $6 \% .^{11}$ Sillay et al. described 420 DBS patients, 19 (4.5\%) of whom developed hardware-related infections requiring reoperation within 6 months. All 19 were treated with partial or complete removal of the involved hardware and antibiotics. ${ }^{38}$

We had 3 infections in our series, with one of the patients not having reached the 1-year posttreatment mark. It is important to stress that the pediatric epilepsy population, with its concomitant comorbidities such as autism, severe anxiety, and obsessive-compulsive disorder, may be at higher risk for wound complications. One of the 3 infections was likely attributable to the patient's continued manual picking of the incision. Careful postoperative wound care is advised in all young patients. We instituted a postoperative paradigm including protection of the incision with dry bandages until postoperative day 5, after which the patient and the family were encouraged to wash the head with shampoo. We maintained frequent communication with the family and instituted the secure sending of photographs of the incision. Two of the 3 infections occurred early in our series and only 1 in the last 36 months. We believe that increasing the comfort with the procedure may aid in decreasing the infection rate. We recommend at least 1 year of follow-up after RNS device implantation to monitor for delayed infections, as seen in one of our patients. Repeat operations to replace end-of-life neurostimulators are a significant burden on the patient and carry an additional risk of infection. While no increased risk from repeat revisions was found, ${ }^{39}$ decreasing the lifetime number of device replacements seems a valuable way to decrease the absolute infection risk. The newly available neurostimulator model 320 doubles the battery life from 4 to 8 years, thus lowering the number of replacements over the lifespan of the patient. To date, no infections have occurred in the 6 replacements in our series.

\section{Failure of Hardware}

Baizabal et al. investigated complications from DBS for Parkinson's disease in 512 patients and found that lead fracture was the most common complication $(2.5 \%) .{ }^{40} \mathrm{In}$ another DBS study by Chan et al., hardware malfunctions were reported as fractures, migration, and erosion of electrodes. ${ }^{41}$ Unlike DBS, RNS bypasses extensions because the distance to the neurostimulator is shorter. ${ }^{42}$ Thus, RNS may have a lower inherent risk of lead fracture. While none were seen in our cohort, larger studies and longer follow-ups would be required to evaluate hardware migration and erosion.

\section{Techniques}

Our experience with pediatric RNS prompts a brief discussion on minimizing complications and streamlining RNS system implantation at centers beginning to use this approach. Concerns about pediatric skull anatomy, including skull thickness and growth, have been raised. While the human skull does not reach adult size until around 7 years old, the growth curve significantly flattens after 3-4 years of age. ${ }^{43}$ While the Back to Sleep campaign has brought about significant plagiocephalic deformity to $20 \%$ of all infants, the approximately 5 -cm RNS device is contoured to the skull and does not alter skull shape or growth. In our series, scalp breakdown was not seen given the mandate to place the device flush with the skull outer table. This finding is significant considering the thin soft tissues of the pediatric scalp. All 3 infections occurred away from the ferrule site at a small depth incision and were not caused by device pressure or skin breakdown. We consider it safe to apply pin fixation and to place the RNS ferrule in patients above 4 years of age, although there is no hard age cutoff. Preoperative CT (low radiation protocol to evaluate bony anatomy) is valuable to assess 
the thickness for pinning, craniotomy design, as well as bone fiducial placement. Although children present unique anatomical challenges because of their smaller, thinner skulls, we did not encounter complications intrinsic to pediatric anatomy during stereotactic head fixation or RNS system implantation. The avoidance of atlas-based targeting in pediatric neuromodulation is generally suggested because of significant anatomical variability, especially in the thalamic architecture from developmental abnormalities and previous surgeries.

The parietal boss appears optimal for ferrule placement given its thickness and location away from suture lines, decreasing the theoretical risk of arrested skull growth. Countersinking the ferrule is crucial in patients with a thin skull and thin skin. We observed that mild pressure on the dura mater is better tolerated than any pressure outward on the healing skin. Bending the "legs" of the ferrule closer to the screw hole allows deeper placement, potentially preventing wound complications (Fig. 1 left).

Direct anatomical targeting in lieu of MER for depth electrodes seems preferable in a pediatric epilepsy cohort. Unlike DBS for parkinsonian, tremor, and dystonia patients, the value of MER in epilepsy is unproven, as the majority of targets do not have agreed upon characteristics. The downsides of MER are significant, including keeping a pediatric patient engaged during a lengthy procedure as well as the additional bleeding risk from multiple MER passes. ${ }^{31,44,45}$

While the number of parenchymal penetrations in our cohort was small, we hypothesize that as the number of implants increases, the intracranial hemorrhage risk will follow the literature on DBS and adult RNS. ${ }^{11,30,31}$ Such risk is low, and RNS safety is likely to continue to improve with ongoing enhancements in the implantation technique.

In $78 \%$ of cases we placed more than two leads in the hope of having easy-to-institute alternatives if the primary detection or stimulation areas proved insufficient. Infection being a significant concern, we wanted to keep the length of possible revision procedures to a minimum. The additional benefit of an electrode placed proactively for later possible connection via a short outpatient procedure outweighed the risks of placement in several instances. Such decisions were made as a group during the MDC while taking into account the likely risk of additional electrode placement versus the possible benefit to the patient, as well as the time point at which the initial stimulation paradigm would be reevaluated by the group, usually 1 year. Such leads were placed and tunneled as usual but were left capped within easy reach from the neurostimulator inlet. Tucking the leads in a small trough between the skull and the long side of the ferrule, held by a dog bone plate, may simplify later procedures to connect the backup leads (Fig. 1 right).

As with all surgeries that require reopening, thoughtful planning of the incision is of the utmost importance. For example, the ferrule must be placed carefully so that only a small opening is sufficient to remove or replace the neurostimulator and to exchange the connected leads. Even with continuing improvements in battery life and the eventual introduction of rechargeable configurations, children are still likely to undergo numerous procedures during their lifetime. While the current data do not show an increased

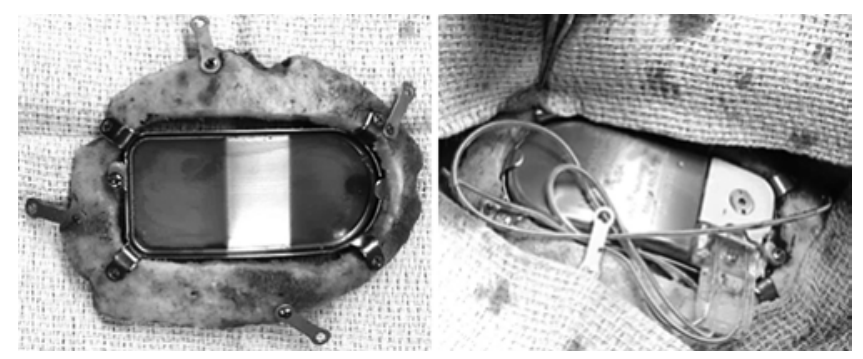

FIG. 1. Techniques for lead placement during RNS system implantation into the skull. Left: To avoid wound problems, the "legs" of the ferrule were bent so that they were closer to the screw hole to allow the ferrule to sit deeply in the skull. Right: A demonstration of the method used to place backup leads into a small trough between the skull and the sides of the ferrule, with application of a dog bone to keep the leads in place. While there is a recommendation to avoid wires over the neurostimulator, the true no-go area is the tan connector cover. In our experience, we have not seen interference with a mild overlap of wires over the metallic casing.

risk for these procedures, ${ }^{39}$ any safeguard to decrease the infection risk is desired.

Many patients undergoing RNS device implantation have had previous neurosurgical interventions. Therefore, using an old craniotomy and fashioning the ferrule within may increase surgical efficiency (Fig. 1 left). It is also possible that the initial surgery failed because of the residual epileptogenic foci at the perimeter of the resection or at nearby eloquent areas or because of the initial quiescence of surrounding tissues during previous diagnostic monitoring. In such cases, reopening the previous craniotomy may save additional incisions and allow for strip or depth electrode placement in concerning areas.

Historically, neuromodulation was borne out of lesioning. There has been a slow paradigm shift in movement disorders evolving from irreversible to reversible and from invasive to minimally invasive. It is possible that we may see the same trajectory for pediatric epilepsy treatment. As neuromodulation continues to increase its share of the market, we may see a philosophical shift in the community, especially in pediatric patients and families. Resections and ablations may decrease or may be put off in favor of a longer evaluation period with chronic implanted ambulatory EEG. While patients are not likely to be seizure free during this time, they are likely to have better control of their condition and the risk of sudden unexpected death in epilepsy (SUDEP) is decreased..$^{11}$ Utilization of chronic recordings may in turn show that resection or ablation is a possibility and may augment the efficacy of such eventual treatment. Further pediatric studies are needed to show the neuropsychological and quality of life benefits of avoiding resection. While already reported in adults, the value of "getting it right" in pediatric patients may be even more important because of their life expectancy and the higher incidence of surgical failure with resective and ablative methods. ${ }^{24}$

\section{Study Limitations}

Although our initial results with pediatric RNS are promising, our study has several limitations that must be addressed. This study is a retrospective single-institution 
experience. To alleviate the former shortcoming, we have proposed to an IRB a prospective analysis of pediatric patients treated with RNS and are working with our hospital legal team on a standardized consent process. To ameliorate the latter limitation (single institution), we are working on partnering with other hospitals to present a multicenter experience. Furthermore, as a large referral center, our institution harbors a potential selection bias for cases of higher complexity. Given our relatively small cohort, a sampling error must be considered as well. Our patients had regular outpatient follow-up visits with the same epileptologists after implantation of the RNS system. During these sessions, some patients had adjustments in their antiepileptic drug regimen to optimize seizure control. Therefore, the observed improvement in outcomes could be partially attributed to medication adjustments. Because of the small sample size and limited follow-up, it is beyond the scope of this safety paper to analyze outcomes in terms of the following variables: response to neurostimulation as stratified by lobe and circuit, evaluation of medication reduction with RNS, and elucidation of response rates related to targeting accuracy of the electrodes. Our center will continue to work on such questions for upcoming publications as the cohort increases in size and the follow-up reaches 3 - and 5-year milestones.

\section{Conclusions}

Our preliminary data suggest that RNS is safe in pediatric patients with DRE, with the greatest risk being postoperative infection. RNS offers a good risk-benefit ratio with the goal of alleviating the seizure burden of patients with medically refractory epilepsy.

\section{Acknowledgments}

We would like to thank Sean Sliger and Hannah Timm from NeuroPace for assisting us with data collection.

\section{References}

1. Hauser WA, Annegers JF, Rocca WA. Descriptive epidemiology of epilepsy: contributions of population-based studies from Rochester, Minnesota. Mayo Clin Proc. 1996;71(6): $576-586$.

2. Wirrell EC. Predicting pharmacoresistance in pediatric epilepsy. Epilepsia. 2013;54(suppl 2):19-22.

3. Harrison RM, Taylor DC. Childhood seizures: a 25-year follow up. Social and medical prognosis. Lancet. 1976;1(7966): 948-951.

4. Dooley J, Gordon K, Camfield P, et al. Discontinuation of anticonvulsant therapy in children free of seizures for 1 year: a prospective study. Neurology. 1996;46(4):969-974.

5. Berg AT, Shinnar S, Levy SR, et al. Defining early seizure outcomes in pediatric epilepsy: the good, the bad and the inbetween. Epilepsy Res. 2001;43(1):75-84.

6. Nair DR. Management of drug-resistant epilepsy. Continuum (Minneap Minn). 2016;22(1 Epilepsy):157-172.

7. Rennert RC, Khan U, Tatter SB, et al. Patterns of clinical use of stereotactic laser ablation: analysis of a multicenter prospective registry. World Neurosurg. 2018;116:e566-e570.

8. Curry DJ, Gowda A, McNichols RJ, Wilfong AA. MR-guided stereotactic laser ablation of epileptogenic foci in children. Epilepsy Behav. 2012;24(4):408-414.

9. McCracken DJ, Willie JT, Fernald BA, et al. Magnetic reso- nance thermometry-guided stereotactic laser ablation of cavernous malformations in drug-resistant epilepsy: imaging and clinical results. Oper Neurosurg (Hagerstown). 2016;12(1): 39-48.

10. Heck CN, King-Stephens D, Massey AD, et al. Two-year seizure reduction in adults with medically intractable partial onset epilepsy treated with responsive neurostimulation: final results of the RNS System pivotal trial. Epilepsia. 2014;55(3): 432-441.

11. Bergey GK, Morrell MJ, Mizrahi EM, et al. Long-term treatment with responsive brain stimulation in adults with refractory partial seizures. Neurology. 2015;84(8):810-817.

12. Pomata HB, González R, Bartuluchi M, et al. Extratemporal epilepsy in children: candidate selection and surgical treatment. Childs Nerv Syst. 2000;16(12):842-850.

13. Starnes K, Miller K, Wong-Kisiel L, Lundstrom BN. A review of neurostimulation for epilepsy in pediatrics. Brain Sci. 2019;9(10):E283.

14. Lee B, Zubair MN, Marquez YD, et al. A single-center experience with the NeuroPace RNS system: a review of techniques and potential problems. World Neurosurg. 2015;84(3): 719-726.

15. Sun FT, Morrell MJ, Wharen RE Jr. Responsive cortical stimulation for the treatment of epilepsy. Neurotherapeutics. 2008;5(1):68-74.

16. Loring DW, Kapur R, Meador KJ, Morrell MJ. Differential neuropsychological outcomes following targeted responsive neurostimulation for partial-onset epilepsy. Epilepsia. 2015; 56(11):1836-1844.

17. Kokoszka MA, Panov F, La Vega-Talbott M, et al. Treatment of medically refractory seizures with responsive neurostimulation: 2 pediatric cases. J Neurosurg Pediatr. 2018;21(4): 421-427.

18. Singhal NS, Numis AL, Lee MB, et al. Responsive neurostimulation for treatment of pediatric drug-resistant epilepsy. Epilepsy Behav Case Rep. 2018;10:21-24.

19. McGovern RA, Alomar S, Bingaman WE, Gonzalez-Martinez J. Robot-assisted responsive neurostimulator system placement in medically intractable epilepsy: instrumentation and technique. Oper Neurosurg (Hagerstown). 2019;16(4): 455-464.

20. Nair D, Morell M. Long-term safety and efficacy of responsive brain stimulation in adults with medically intractable partial onset seizures. Neurology. 2017;88(16)(suppl).

21. DiLorenzo DJ, Mangubat EZ, Rossi MA, Byrne RW. Chronic unlimited recording electrocorticography-guided resective epilepsy surgery: technology-enabled enhanced fidelity in seizure focus localization with improved surgical efficacy. $J$ Neurosurg. 2014;120(6):1402-1414.

22. Warner NM, Gwinn RP, Doherty MJ. Individualizing therapies with responsive epilepsy neurostimulation-a mirtazapine case study of hippocampal excitability. Epilepsy Behav Case Rep. 2016;6:70-72.

23. McGovern RA, Knight EP, Gupta A, et al. Robot-assisted stereoelectroencephalography in children. J Neurosurg Pediatr. 2018;23(3):288-296.

24. Ansari SF, Maher CO, Tubbs RS, et al. Surgery for extratemporal nonlesional epilepsy in children: a meta-analysis. Childs Nerv Syst. 2010;26(7):945-951.

25. Najm I, Jehi L, Palmini A, et al. Temporal patterns and mechanisms of epilepsy surgery failure. Epilepsia. 2013; 54(5):772-782.

26. Gillis J, Loughlan P. Not just small adults: the metaphors of paediatrics. Arch Dis Child. 2007;92(11):946-947.

27. Manjunath R, Paradis PE, Parisé H, et al. Burden of uncontrolled epilepsy in patients requiring an emergency room visit or hospitalization. Neurology. 2012;79(18):1908-1916.

28. Arya R, Mangano FT, Horn PS, et al. Adverse events related to extraoperative invasive EEG monitoring with subdural 
grid electrodes: a systematic review and meta-analysis. Epilepsia. 2013;54(5):828-839.

29. DeLong MR, Huang KT, Gallis J, et al. Effect of advancing age on outcomes of deep brain stimulation for Parkinson disease. JAMA Neurol. 2014;71(10):1290-1295.

30. Seijo F, Alvarez de Eulate Beramendi S, Santamarta Liébana E, et al. Surgical adverse events of deep brain stimulation in the subthalamic nucleus of patients with Parkinson's disease. The learning curve and the pitfalls. Acta Neurochir (Wien). 2014;156(8):1505-1512.

31. Zrinzo L, Foltynie T, Limousin P, Hariz MI. Reducing hemorrhagic complications in functional neurosurgery: a large case series and systematic literature review. J Neurosurg. 2012;116(1):84-94.

32. Starr PA, Markun LC, Larson PS, et al. Interventional MRIguided deep brain stimulation in pediatric dystonia: first experience with the ClearPoint system. J Neurosurg Pediatr. 2014;14(4):400-408.

33. Musleh W, Yassari R, Hecox K, et al. Low incidence of subdural grid-related complications in prolonged pediatric EEG monitoring. Pediatr Neurosurg. 2006;42(5):284-287.

34. Johnston JM Jr, Mangano FT, Ojemann JG, et al. Complications of invasive subdural electrode monitoring at St. Louis Children's Hospital, 1994-2005. J Neurosurg. 2006;105(5) (suppl):343-347.

35. Murphy JV, Torkelson R, Dowler I, et al. Vagal nerve stimulation in refractory epilepsy: the first 100 patients receiving vagal nerve stimulation at a pediatric epilepsy center. Arch Pediatr Adolesc Med. 2003;157(6):560-564.

36. Smyth MD, Tubbs RS, Bebin EM, et al. Complications of chronic vagus nerve stimulation for epilepsy in children. $J$ Neurosurg. 2003;99(3):500-503.

37. Salanova V, Witt T, Worth R, et al. Long-term efficacy and safety of thalamic stimulation for drug-resistant partial epilepsy. Neurology. 2015;84(10):1017-1025.

38. Sillay KA, Larson PS, Starr PA. Deep brain stimulator hardware-related infections: incidence and management in a large series. Neurosurgery. 2008;62(2):360-367.

39. Weber PB, Kapur R, Gwinn RP, et al. Infection and erosion rates in trials of a cranially implanted neurostimulator do not increase with subsequent neurostimulator placements. Stereotact Funct Neurosurg. 2017;95(5):325-329.

40. Baizabal Carvallo JF, Mostile G, Almaguer M, et al. Deep brain stimulation hardware complications in patients with movement disorders: risk factors and clinical correlations. Stereotact Funct Neurosurg. 2012;90(5):300-306.
41. Chan DT, Zhu XL, Yeung JH, et al. Complications of deep brain stimulation: a collective review. Asian J Surg. 2009; 32(4):258-263.

42. Miller PM, Gross RE. Wire tethering or 'bowstringing' as a long-term hardware-related complication of deep brain stimulation. Stereotact Funct Neurosurg. 2009;87(6):353-359.

43. Frassanito P, Bianchi F, Pennisi G, et al. The growth of the neurocranium: literature review and implications in cranial repair. Childs Nerv Syst. 2019;35(9):1459-1465.

44. Hariz MI. Surgical probings into the basal ganglia: Hemorrhage and hardware-related risks, and costs of microelectrode recording. Mov Disord. 2011;26(8):1375-1377.

45. Hariz MI. Safety and risk of microelectrode recording in surgery for movement disorders. Stereotact Funct Neurosurg. 2002;78(3-4):146-157.

\section{Disclosures}

Drs. Ghatan and Panov are consultants for NeuroPace. Dr. Wolf has received clinical or research support from NeuroPace for the study described.

\section{Author Contributions}

Conception and design: Panov, Haskell, Fields, La Vega-Talbott, Wolf, McGoldrick, Marcuse, Ghatan. Acquisition of data: all authors. Analysis and interpretation of data: all authors. Drafting the article: all authors. Critically revising the article: all authors. Reviewed submitted version of manuscript: all authors. Approved the final version of the manuscript on behalf of all authors: Panov. Statistical analysis: Panov, Fields, La Vega-Talbott, Wolf, McGoldrick, Marcuse, Ghatan. Administrative/technical/ material support: Panov, Fields, La Vega-Talbott, Wolf, McGoldrick, Marcuse, Ghatan. Study supervision: Panov, Fields, La Vega-Talbott, Wolf, McGoldrick, Marcuse, Ghatan.

\section{Correspondence}

Fedor Panov: Mount Sinai West, New York, NY. fedor.panov@ mountsinai.org. 